sto

3 Research Square

Preprints are preliminary reports that have not undergone peer review.

They should not be considered conclusive, used to inform clinical practice, or referenced by the media as validated information.

\title{
Understanding the Impact of Patients' Disease Types on Caregiving Time and Caregiver Burden: An Analysis of the Health Information National Trends Survey
}

\author{
Zhaohui Su ( $\nabla$ szh@utexas.edu ) \\ University of Texas Health Science Center at San Antonio https://orcid.org/0000-0003-2005-9504 \\ Zi Zhou \\ Xiamen University \\ Jonathan Gelfond \\ University of Texas Health Science Center at San Antonio
}

\section{Research article}

Keywords: patients' disease types; caregiving time; caregiver burden; HINTS; secondary data analysis

Posted Date: August 4th, 2020

DOI: https://doi.org/10.21203/rs.3.rs-48085/v1

License: (9) (1) This work is licensed under a Creative Commons Attribution 4.0 International License. Read Full License 


\section{Abstract}

Background: Patients with different medical conditions often have distinctive caregiving needs that could result in varying levels of caregiver burden. However, despite empirical advances in this area, little is known about how patients' disease types interact with caregiving time and caregiver burden. To bridge this gap, we examined the impact of patients' disease types on caregiving time and burden.

Methods: Data were analyzed from the 2018 Health Information National Trends Survey 5 Cycle 2. Only participants self-identified as caregivers were included in the final analysis. Data on patients' disease types, caregiving time (i.e., caregiving duration and caregiving hours spent per week), and caregiver burden (i.e., caregivers' self-rated health, body mass index, and psychological distress) were examined using logistic regression analysis.

Results: Patients' disease types impacted caregiving time and burden. Caregivers of patients with neurological disease spent the greatest amount of time. For caregiver burden, caregivers of patients with cancer or aging related disease experienced worst self-rated health, caregivers of patients with orthopedic disease have the greatest likelihood to be overweight or obese, while cancer caregivers experienced greatest levels of psychological distress.

Conclusions: Patients' disease types had highly varied effects on caregiving time and burden. This study underscores the need for healthcare researchers to adopt a nuanced approach in acknowledging and addressing the burden of care experienced by caregivers, such as tailoring interventions based on both patients and caregivers' characteristics and preferences.

\section{Introduction}

Caregiving is a challenging experience that often results in physical and psychological burden on informal caregivers [1-3]. An informal caregiver is a person who offers unpaid or ill-compensated care to a family member, friend, or partner due to illness-related reasons or old age. Results indicate that informal caregivers often experience substantial physical and psychological health consequences as a result of caregiving, such as fatigue [4,5], loss of sleep [6, 7], perceived stigma [8, 9], anxiety symptoms [10, 11], depression disorders [12, 13], worsened subjective wellbeing $[14,15]$, and compromised quality of life [16, 17]. Evidence further shows that informal caregivers' selfrated health has been in decline for the past five years, further widening the gap between caregivers and the general population' health status [18]. As more than one in five Americans ( 53 million) is an informal caregiver [18], research is needed to better understand factors that influence caregiver burden so that more effective health solutions could be developed to alleviate resultant heath disparities.

Across disease contexts, factors that may impact caregiver burden have been examined, including caregivers' sociodemographic characteristics (e.g., gender) [19], caregiver self-care abilities (e.g., self-efficacy) [20], social support factors (e.g., caregiving unmet needs) [21], and patient-related factors (e.g., time spent on caregiving) [22]. While less studied, many thanks to the increasing recognition of the interactive nature of caregiving, research on patient-related factors' impact on caregiver burden is gaining momentum [23, 24]. A growing body of evidence suggests that patients' disease types could have a considerable impact on caregiving time and caregiver burden [25-29]. Comparing caregivers of patients face renal transplantation and caregivers of patients undergo hemodialysis, researchers found that caregivers of patients receive hemodialysis had significantly higher levels of caregiver burden [26]. Findings further indicate that caregivers of frontotemporal lobar degeneration and caregivers of dementia with Lewy bodies patients experienced significantly more burden than caregivers of people with Alzheimer's [28]. 
Conflicting findings are also present in the literature [24,30]. In a comparative study, researchers found no significant difference in levels of burden reported by leukemia caregivers and cerebral palsy caregivers [24]. Comparing the impact of Alzheimer's disease and frontotemporal dementia on caregiver burden, study also indicates that there are no significant differences in levels of burden experienced in these two groups of caregivers [31]. Though evidence is needed to clarify mixed findings in the literature, research is scarce. Overall, only a handful of studies have investigated the impact of patients' disease types on caregiving consequences such as caregiving time and caregiver burden $[26,28,29,31,32]$. Furthermore, considering that most of the available studies are either conducted in the cognitive impairment disease context (e.g., comparing different types of dementia conditions) $[28,29]$ or only examined two to three types of distinctive medical conditions' in the analysis [26, 31, 32], these studies are limited in the diversity of insights they can offer. To bridge this gap, we aim to examine the impact of patient's disease types on caregiving time (i.e., caregiving duration and caregiving hours spent per week) and caregiver burden (i.e., caregivers' self-rated health, body mass index or BMI, and psychological distress), using data that examined a comprehensive list of patients' disease types (see Table 1).

Table 1

List of patient's disease types

\section{Disease type Example}

Cancer Skin cancer, breast cancer, lung cancer, etc.

Alzheimer's or Alzheimer's, confusion, dementia (e.g., frontotemporal dementia), forgetfulness.

related issues

Orthopedic or Back problems, broken bones, arthritis, mobility problems, can't get around, feeble, unsteady, related issues falling).

Mental health Mental illness, emotional problems, depression, anxiety, and substance/drug/alcohol abuse. or related

issues

Chronic conditions

Neurological or related issues

Acute conditions

Aging or related issues

Multiple disease conditions

Other conditions

Unknown conditions
High blood pressure/hypertension, diabetes, heart disease, heart attack, lung disease, emphysema, Chronic Obstructive Pulmonary Disease (COPD), Parkinson's

Brain damage or injury, developmental or intellectual disorder, mental retardation, Down syndrome, and stroke.

Health conditions that are severe and sudden in onset, such as a broken bone or an asthma attack.

Aging and related health issues not listed in the other categories above.

We coded this variable to represent patients with multiple health conditions mentioned above or different patients caregivers that have two or more distinctive conditions.

Medical conditions that are not listed above.

Conditions that are unknown to the caregiver and/or patients.

\section{Methods}

\section{Study Design and Participants}


This is a cross-sectional study using data from the Health Information National Trends Survey (HINTS, 2018) Version 5 , Cycle 2 , a nationally representative survey of U.S. adults aged $\geq 18$ years (civilian and non-institutionalized) [33]. The survey is first conducted by the National Cancer Institute ( $\mathrm{NCl})$ in 2003, aiming to gauge how Americans seek, share, and use cancer-related health information in their daily interactions [34]. The HINTS 5 (Cycle 2) survey used in this study was administered from January 26 to May 2, 2018. We utilized data from the 2018 HINTS survey because, by far, caregiver burden related variables were only included in this particular HINTS survey. Of all the 14,585 surveys mailed, 3,527 participants returned their questionnaires (response rate: $24.2 \%$ ) [34]. As this study mainly focuses on the caregiver population, we used "Are you currently caring for or making health care decisions for someone with a medical, behavioral, disability, or other condition?" as the screening question to identify caregivers. A total of 458 (13.0\%) participants who self-identified as caregivers (i.e., replied "yes" to the question) were included in the final data analysis.

\section{Measures}

To adequately gauge the impact of patients' disease types on caregiving consequences, both direct and indirect consequences were evaluated in this study. Based on insights gained from the literature [35-38], direct consequence of caregiving is measured by caregiving time, in terms of caregiving duration and caregiving hours spent per week, while indirect consequences of caregiving are measured by caregiver burden in terms of caregivers' self-rated health, $\mathrm{BMI}$, and psychological distress. Details of all measures used in this study are reported by the National Cancer Institute and available online [34].

\section{Caregiving time}

We adopted two measures to gauge caregivers' time spent on providing care to patients: caregiving duration and caregiving hours spent per week [34].

Caregiving duration. Participants were directed to "Think about the individual for whom you are currently providing the most care. About how long have you been providing care for this person?" and asked to choose one response from the 5 available choices (i.e., "Less than 30 days" $=1$, " 1 to 6 months" = 2, " 7 months to 2 years" = 3 , " 3 to 5 years" $=4$, "More than 5 years" $=5$ ).

Caregiving hours spent per week. Participants were asked to "Think about the individual for whom you are currently providing the most care. About how many hours per week do you spend in an average week providing care?" and indicate hours they spent per week on offering care to the patients.

\section{Caregiver burden}

In line with the literature [35-38], caregiver burden was gauged using three variables: Self-rated health, BMI, and psychological distress.

Self-rated health. To assess respondents' self-rated health status, participants' were asked to respond to the question "In general, would you say your health is. .. " on a five-point scale ("excellent" = 1, "very good" = 2, "good" = 3, "fair" = 4, "poor" = 5).

BMI. Participants' BMI levels were calculated using information on participants' self-rated weight and height (formula: weight $(\mathrm{kg}) /[\text { height }(\mathrm{m})]^{2}$ ). Based on Centers for Disease Control and Prevention's guidelines, BMI was subsequently coded into three categories: underweight or normal (<25), overweight (25-29.9), and obese ( $\geq 30)$ [39]. 
Psychological distress. Participants' psychological distress was measured with the four-item Patient Health Questionnaire (PHQ-4) [34, 40]. Participants completed four items in response to "Over the past 2 weeks, how often have you been bothered by any of the following problems?": (a) Little Interest, (b) hopeless, (c) nervous, (d) worrying on scales from 0 (Not at all) to 3 (nearly every day). Based on available guidelines, the four items were subsequently summed $[34,40]$. The summed scores ranged from 0 to 12, with a higher score indicating a higher level of psychological distress [40].

\section{Caregiver Characteristics}

Demographic factors included were gender ( "male" = 0 vs. "female" = 1), race/ethnicity ("non-Hispanic White" = 1, "other" = 0), and marital status ("married" or "living as married" = 1 vs. "divorced," "widowed," "separated," or "single, never been married" = 0). Other covariates examined were household income ("US\$50,000 or more" $=1$, or "less than US\$50,000/year" = 0), education (coded into four categories: less than high school, high school graduate, some college, and college graduate or more), and employment status ("employed" = 1 vs. "unemployed," "homemaker," "student," "retired," "disabled," or "other" = 0). A 9-point rural urban continuum code was used to classify participants as residing either in metropolitan (codes 1-3) or nonmetropolitan (codes 4-9) areas [41]. Smoking status (categorized as current smoker, former smoker, or never smoker). Drinking status ("having at least one drink of any alcoholic beverage more than 1 day per week" $=1$, "none" $=0$ ). We reclassified the response options into a single dichotomous outcome variable for physical activity, that is, whether the subject (1) met physical activity recommendations ( $\geq 150$ minutes per week) or did not meet the physical activity recommendations ( $<150$ minutes per week). Having health insurance ("yes" = 1, "not" = 0).

\section{Data Analyses}

First, the characteristics of the participants were described using descriptive statistics (means accompanied with SDs or frequencies, as appropriate). Second, to explore the relationship between health outcome and independent variables, we used multivariate logistic regression method for analyzing the self-rated health variable and ordered logistic regressions for the $\mathrm{BMI}$ and psychological distress variables. In order to test the potential interaction, we generated several interaction terms by multiplying the categorical variables. Third, we calculated the mean scores of health indicators and caregiving burden of caregivers. In order to adjust for HINTS' multistage probability sampling design, a set of 50 jackknife replicate weights was applied to all analyses, estimating the model parameters for the U.S. population as a whole. Finally, to explore the association between caregiver burden, caregiver health outcomes, and independent variables, we used ordered logistic regression for analyzing self-rated health, BMI, psychological distress and applied linear regression analyses to examine caregiving duration and caregiving hours spent per week to answer the research question.

Odds ratios (ORs) or regression coefficients with $\mathrm{Cl}$ were employed to depict the relationships between caregiver burden, caregiver health outcomes, and independent variables while controlling for covariates. Listwise deletion of each subjects were used for participants who provided invalid or missing responses for the dependent variables; sample sizes for each regression analysis are noted in Table 3 ranging from 3,256 to 3,264. All covariates with missing data were multiple imputed. All analyses were performed using Stata 14 [42] .

\section{Results}

The characteristics of participants who completed the HINTS questionnaire were illustrated in Table 3. Analyses show that compared to non-caregivers, caregivers experienced higher $\mathrm{BMI}(\mathrm{OR}=1.42,95 \% \mathrm{Cl}=1.18-1.71)$ and greater levels of psychological distress $(\mathrm{OR}=1.44,95 \% \mathrm{Cl}=1.21-1.73)$. This association was statistically significant 
even after controlling for the interaction between caregiver and gender $(\mathrm{OR}=1.96,95 \% \mathrm{Cl}=1.45-2.65)$ and the interaction between caregiver and employment status $(\mathrm{OR}=1.89,95 \% \mathrm{Cl}=1.45-2.47)$. 
Table 2

Characteristics of participants $(\mathrm{N}=3504)$

\begin{tabular}{|c|c|c|c|c|c|}
\hline Variables & Freq. & Percent & Variables & Freq. & Percent \\
\hline Age & & & Employment status & & \\
\hline$\sim 45$ & 843 & 24.06 & Unemployed & 1,761 & 50.84 \\
\hline $45 \sim$ & 1,396 & 39.84 & Employed & 1,703 & 49.16 \\
\hline $65 \sim$ & 1,265 & 36.1 & Smoking & & \\
\hline Sex & & & Current & 460 & 13.13 \\
\hline Male & 1,416 & 40.41 & Former & 880 & 25.11 \\
\hline Female & 2,088 & 59.59 & Never & 2,164 & 61.76 \\
\hline Race & & & Drinking & & \\
\hline Others & 1,328 & 37.9 & Not & 2,086 & 59.53 \\
\hline Non-Hispanic White & 2,176 & 62.1 & Yes & 1,418 & 40.47 \\
\hline Residence & & & Recommended PA* & & \\
\hline Non-metropolitan & 489 & 13.96 & Not & 2,323 & 66.3 \\
\hline Metropolitan & 3,015 & 86.04 & Yes & 1,181 & 33.7 \\
\hline Marital status & & & Having Health Insuran & & \\
\hline Unmarried & 1,737 & 49.57 & Not & 248 & 7.08 \\
\hline Married & 1,767 & 50.43 & Yes & 3,256 & 92.92 \\
\hline Educational level & & & Emotional Support & & \\
\hline Less than $\mathrm{HS}^{\#}$ & 281 & 8.02 & Never & 140 & 4.00 \\
\hline HS Grad & 643 & 18.35 & Rarely & 194 & 5.54 \\
\hline Some College & 1,050 & 29.97 & Sometimes & 483 & 13.78 \\
\hline College Graduate & 1,530 & 43.66 & Often & 554 & 15.81 \\
\hline Income Ranges & & & Always & 2,133 & 60.87 \\
\hline$\$ 0$ to $\$ 9,999$ & 245 & 7.00 & Self-care Efficacy & & \\
\hline$\$ 10,000$ to $\$ 14,999$ & 212 & 6.05 & Completely confident & 33 & 0.95 \\
\hline$\$ 15,000$ to $\$ 19,999$ & 198 & 5.65 & Very confident & 110 & 3.17 \\
\hline$\$ 20,000$ to $\$ 34,999$ & 484 & 13.81 & Somewhat confident & 834 & 24.03 \\
\hline$\$ 35,000$ to $\$ 49,999$ & 458 & 13.07 & A little confident & 1,618 & 46.61 \\
\hline$\$ 50,000$ to $\$ 74,999$ & 631 & 18.01 & Not confident at all & 876 & 25.24 \\
\hline$\$ 75,000$ to $\$ 99,999$ & 412 & 11.76 & Caregiver & & \\
\hline
\end{tabular}




\begin{tabular}{|llllll|}
\hline Variables & Freq. & Percent & Variables & Freq. & Percent \\
\hline$\$ 100,000$ to $\$ 19,999$ & 635 & 18.12 & Not & 2,918 & 85.77 \\
\hline 200,000 or more & 229 & 6.54 & Yes & 484 & 14.23 \\
\hline Freq.=frequency; *PA = physical activity; ${ }^{*} \mathrm{HS}=$ high school & & \\
\hline
\end{tabular}


Table 3

Factors associated with general health, $\mathrm{BMI}$, and psychological distress

\begin{tabular}{|c|c|c|c|c|c|}
\hline \multirow[t]{2}{*}{ Variables } & \multirow{2}{*}{$\begin{array}{l}\text { General } \\
\text { Health } \\
\text { (1) }\end{array}$} & \multirow{2}{*}{$\begin{array}{l}\text { BMI3 } \\
\text { (2) }\end{array}$} & \multicolumn{3}{|c|}{ Psychological Distress } \\
\hline & & & (3) & (4) & \\
\hline \multirow[t]{2}{*}{ Caregiver (Ref: Not) } & 1.22 & $1.42^{\star \star \star}$ & $1.44^{\star \star \star}$ & $1.96 * \star \star$ & $1.89 * \star \star$ \\
\hline & $(0.89-1.66)$ & $\begin{array}{l}(1.18- \\
1.71)\end{array}$ & $\begin{array}{l}(1.21- \\
1.73)\end{array}$ & $\begin{array}{l}(1.45- \\
2.65)\end{array}$ & $\begin{array}{l}(1.45- \\
2.47)\end{array}$ \\
\hline \multirow[t]{2}{*}{ Self-care efficacy } & $0.22 * \star \star$ & $0.72^{\star \star *}$ & $0.51 * \star \star$ & $0.51 * * *$ & $0.51 * * *$ \\
\hline & $(0.18-0.26)$ & $\begin{array}{l}(0.66- \\
0.79)\end{array}$ & $\begin{array}{l}(0.47- \\
0.56)\end{array}$ & $\begin{array}{l}(0.47- \\
0.56)\end{array}$ & $\begin{array}{l}(0.47- \\
0.56)\end{array}$ \\
\hline \multirow{2}{*}{$\begin{array}{l}\text { Metropolitan (Ref: Non-metropolitan } \\
\text { ) }\end{array}$} & 0.78 & 0.83 & 1.00 & 1.01 & 1.01 \\
\hline & $(0.58-1.05)$ & $\begin{array}{l}(0.68- \\
1.01)\end{array}$ & $\begin{array}{l}(0.82- \\
1.22)\end{array}$ & $\begin{array}{l}(0.83- \\
1.23)\end{array}$ & $\begin{array}{l}(0.83- \\
1.23)\end{array}$ \\
\hline \multirow[t]{2}{*}{ Age } & $1.02^{\star \star \star}$ & $1.01^{\star}$ & $0.98 * \star \star$ & $0.98 * \star \star$ & $0.98 * \star *$ \\
\hline & $(1.01-1.03)$ & $\begin{array}{l}(1.00- \\
1.01)\end{array}$ & $\begin{array}{l}(0.97- \\
0.98)\end{array}$ & $\begin{array}{l}(0.97- \\
0.98)\end{array}$ & $\begin{array}{l}(0.97- \\
0.98)\end{array}$ \\
\hline \multirow[t]{2}{*}{ Female (Ref: male) } & 1.08 & $0.79 * \star \star$ & $1.79 * \star \star$ & $1.92^{\star \star \star}$ & $1.76^{\star \star \star}$ \\
\hline & $(0.86-1.36)$ & $\begin{array}{l}(0.69- \\
0.91)\end{array}$ & $\begin{array}{l}(1.56- \\
2.05)\end{array}$ & $\begin{array}{l}(1.65- \\
2.23)\end{array}$ & $\begin{array}{l}(1.53- \\
2.02)\end{array}$ \\
\hline \multirow[t]{2}{*}{ Non-Hispanic White (Ref: Others) } & $0.64^{\star * \star}$ & $0.86^{\star}$ & $1.41^{\star \star \star}$ & $1.42^{\star \star \star}$ & $1.40 * \star \star$ \\
\hline & $(0.50-0.82)$ & $\begin{array}{l}(0.74- \\
0.99)\end{array}$ & $\begin{array}{l}(1.21- \\
1.63)\end{array}$ & $\begin{array}{l}(1.23- \\
1.65)\end{array}$ & $\begin{array}{l}(1.20- \\
1.62)\end{array}$ \\
\hline \multirow[t]{2}{*}{ Married (Ref: Not married) } & 1.03 & $1.17^{\star}$ & $0.79 * *$ & $0.79 \star \star$ & $0.78^{\star * *}$ \\
\hline & $(0.82-1.29)$ & $\begin{array}{l}(1.00- \\
1.35)\end{array}$ & $\begin{array}{l}(0.68- \\
0.92)\end{array}$ & $\begin{array}{l}(0.68- \\
0.91)\end{array}$ & $\begin{array}{l}(0.67- \\
0.90)\end{array}$ \\
\hline \multirow[t]{2}{*}{ Education } & $0.80 \star \star$ & $0.74^{\star \star \star}$ & $1.10^{*}$ & $1.10 *$ & $1.10^{*}$ \\
\hline & $(0.70-0.92)$ & $\begin{array}{l}(0.68- \\
0.80)\end{array}$ & $\begin{array}{l}(1.01- \\
1.20)\end{array}$ & $\begin{array}{l}(1.01- \\
1.19)\end{array}$ & $\begin{array}{l}(1.01- \\
1.20)\end{array}$ \\
\hline \multirow[t]{2}{*}{ Employed (Ref: Not employed) } & 0.83 & $1.50 * \star \star$ & $0.73^{\star * *}$ & $0.72 * \star \star$ & $0.80 \star \star$ \\
\hline & $(0.64-1.07)$ & $\begin{array}{l}(1.29- \\
1.75)\end{array}$ & $\begin{array}{l}(0.63- \\
0.85)\end{array}$ & $\begin{array}{l}(0.62- \\
0.84)\end{array}$ & $\begin{array}{l}(0.68- \\
0.94)\end{array}$ \\
\hline \multirow[t]{2}{*}{ Income Ranges } & $0.93^{*}$ & 1.01 & $0.88^{\star \star \star}$ & $0.88^{\star * *}$ & $0.88 * \star \star$ \\
\hline & $(0.87-1.00)$ & $\begin{array}{l}(0.97- \\
1.04)\end{array}$ & $\begin{array}{l}(0.85- \\
0.91)\end{array}$ & $\begin{array}{l}(0.85- \\
0.91)\end{array}$ & $\begin{array}{l}(0.85- \\
0.91)\end{array}$ \\
\hline \multicolumn{6}{|l|}{ BMI (Ref: Normal) } \\
\hline \multirow[t]{2}{*}{ Overweight } & $1.40 *$ & - & $1.24 *$ & $1.26 \star \star$ & $1.26 * \star$ \\
\hline & $(1.04-1.87)$ & & $\begin{array}{l}(1.05- \\
1.47)\end{array}$ & $\begin{array}{l}(1.06- \\
1.49)\end{array}$ & $\begin{array}{l}(1.06- \\
1.49)\end{array}$ \\
\hline
\end{tabular}




\begin{tabular}{|c|c|c|c|c|c|}
\hline \multirow[t]{2}{*}{ Variables } & \multirow{2}{*}{$\begin{array}{l}\text { General } \\
\text { Health } \\
(1)\end{array}$} & \multirow{2}{*}{$\begin{array}{l}\text { BMI3 } \\
(2)\end{array}$} & \multicolumn{3}{|c|}{ Psychological Distress } \\
\hline & & & (3) & (4) & \\
\hline \multirow[t]{2}{*}{ Obesity } & $2.30^{\star \star \star \star}$ & - & 1.15 & 1.15 & 1.15 \\
\hline & $(1.74-3.04)$ & & $\begin{array}{l}(0.97- \\
1.36)\end{array}$ & $\begin{array}{l}(0.97- \\
1.37)\end{array}$ & $\begin{array}{l}(0.97- \\
1.36)\end{array}$ \\
\hline \multicolumn{6}{|l|}{ Smoking (Ref: Current smoker) } \\
\hline \multirow[t]{2}{*}{ Former } & 0.70 & 1.22 & $0.77^{\star}$ & $0.77^{*}$ & $0.77^{*}$ \\
\hline & $(0.48-1.04)$ & $\begin{array}{l}(0.97- \\
1.53)\end{array}$ & $\begin{array}{l}(0.61- \\
0.97)\end{array}$ & $\begin{array}{l}(0.61- \\
0.97)\end{array}$ & $\begin{array}{l}(0.61- \\
0.96)\end{array}$ \\
\hline \multirow[t]{2}{*}{ Never } & $0.58 * \star$ & 1.07 & $0.67^{\star \star \star}$ & $0.67 * \star \star$ & $0.67^{\star \star \star}$ \\
\hline & $(0.40-0.83)$ & $\begin{array}{l}(0.88- \\
1.30)\end{array}$ & $\begin{array}{l}(0.55- \\
0.81)\end{array}$ & $\begin{array}{l}(0.55- \\
0.81)\end{array}$ & $\begin{array}{l}(0.55- \\
0.81)\end{array}$ \\
\hline \multirow[t]{2}{*}{ Drinking (Ref: Not) } & $0.65^{\star \star \star}$ & $0.74^{\star \star \star}$ & 1.11 & 1.11 & 1.11 \\
\hline & $(0.51-0.82)$ & $\begin{array}{l}(0.64- \\
0.85)\end{array}$ & $\begin{array}{l}(0.97- \\
1.28)\end{array}$ & $\begin{array}{l}(0.97- \\
1.28)\end{array}$ & $\begin{array}{l}(0.97- \\
1.28)\end{array}$ \\
\hline \multirow{3}{*}{$\begin{array}{l}\text { Physical Activity } \\
\text { (Ref: }<150 \text { minutes per week) }\end{array}$} & $0.72^{\star \star}$ & $0.65^{\star \star \star}$ & 0.90 & 0.90 & 0.89 \\
\hline & & & & & \\
\hline & $(0.57-0.90)$ & $\begin{array}{l}(0.56- \\
0.74)\end{array}$ & $\begin{array}{l}(0.78- \\
1.05)\end{array}$ & $\begin{array}{l}(0.78- \\
1.05)\end{array}$ & $\begin{array}{l}(0.77- \\
1.04)\end{array}$ \\
\hline \multirow[t]{2}{*}{ Having Health Insurance (Ref: not) } & 1.50 & 1.07 & 0.95 & 0.95 & 0.95 \\
\hline & $(0.90-2.51)$ & $\begin{array}{l}(0.85- \\
1.34)\end{array}$ & $\begin{array}{l}(0.75- \\
1.19)\end{array}$ & $\begin{array}{l}(0.75- \\
1.20)\end{array}$ & $\begin{array}{l}(0.76- \\
1.20)\end{array}$ \\
\hline \multirow[t]{2}{*}{ Emotional Support } & 1.06 & 0.97 & $0.81^{\star \star \star}$ & $0.81 * \star \star$ & $0.81^{\star \star \star}$ \\
\hline & $(0.94-1.19)$ & $\begin{array}{l}(0.91- \\
1.04)\end{array}$ & $\begin{array}{l}(0.76- \\
0.87)\end{array}$ & $\begin{array}{l}(0.76- \\
0.87)\end{array}$ & $\begin{array}{l}(0.76- \\
0.87)\end{array}$ \\
\hline \multirow[t]{2}{*}{ Psychological Distress } & $1.15^{\star \star \star}$ & 1.02 & - & - & - \\
\hline & $(1.10-1.20)$ & $\begin{array}{l}(1.00- \\
1.05)\end{array}$ & & & \\
\hline \multirow[t]{2}{*}{ Caregiver $\times$ Female } & & & & $0.63^{*}$ & \\
\hline & & & & $\begin{array}{l}(0.43- \\
0.91)\end{array}$ & \\
\hline \multirow[t]{2}{*}{ Caregiver $\times$ Employed } & & & & & $0.61^{\star \star}$ \\
\hline & & & & & $\begin{array}{l}(0.43- \\
0.88)\end{array}$ \\
\hline Observations & 3,264 & 3,264 & 3,264 & 3,264 & 3,264 \\
\hline
\end{tabular}


Results on factors associated with self-rated health, BMI, and psychological distress were presented in Table 2. Both the interaction effect between caregiver and gender $(\mathrm{OR}=0.63,95 \% \mathrm{Cl}=0.43-0.91)$ and the interaction effect between caregiver and employment status $(\mathrm{OR}=0.61,95 \% \mathrm{Cl}=0.43-0.88)$ were statistically significant. That is, caregivers experienced greater psychological distress for men than women, and unemployed caregivers experienced greater psychological distress than employed caregivers.

As presented in Table 4, caregivers who were female, older, had lower levels of education, SES status, physical inactivity, unmarried, unemployed, not having health insurance experienced greatest caregiver burden and worst health outcomes. Patients' disease types significantly influenced caregiving time and caregiver burden. In terms of time caregiving hours spent weekly, caregivers of patients with neurological or related conditions spent the greatest amount of time in caregiving (44.6 hours per week), followed by caregivers undertake multiple disease conditions (32.3 h), chronic conditions (32.0 h), unknown disease conditions (31.7 h), cancer (31.1 h), orthopedic or related conditions (29.5 h), Alzheimer's or related conditions (27.1 h), other disease conditions (22.7 h), mental health or related conditions (17.34), aging or aging related conditions (14.9 h), and acute conditions (12.9 h). 
Table 4

The characteristics and the mean scores of general health, $\mathrm{BMI}$, and psychological distress, caregiving duration, and hours per week of caregivers

\begin{tabular}{|c|c|c|c|c|c|c|c|}
\hline Variables & Freq. & Percent & $\mathrm{SRH}^{*}$ & BMI* & $\begin{array}{l}\text { Psychological } \\
\text { distress }\end{array}$ & $\begin{array}{l}\text { Length of } \\
\text { caregiving* }\end{array}$ & $\begin{array}{l}\text { Caregiving } \\
\text { hours/week* }\end{array}$ \\
\hline \multicolumn{8}{|l|}{ Patients' Disease Types } \\
\hline Aging/Aging related & 16 & 3.37 & 2.81 & 1.19 & 3.40 & 3.56 & 14.86 \\
\hline Cancer & 10 & 2.11 & 2.80 & 1.20 & 4.20 & 2.80 & 31.14 \\
\hline Orthopedic/Musculoskeletal & 17 & 3.58 & 2.71 & 1.35 & 3.18 & 4.00 & 29.50 \\
\hline Alzheimer's and related & 30 & 6.32 & 2.70 & 1.13 & 1.73 & 3.55 & 27.08 \\
\hline Multiple disease conditions & 253 & 53.26 & 2.60 & 1.20 & 2.25 & 3.93 & 32.30 \\
\hline Mental health and related & 36 & 7.58 & 2.58 & 1.19 & 2.85 & 4.24 & 17.34 \\
\hline Chronic conditions & 28 & 5.89 & 2.50 & 1.18 & 2.39 & 4.26 & 32.04 \\
\hline Neurological or related & 21 & 4.42 & 2.43 & 1.19 & 1.14 & 4.81 & 44.57 \\
\hline Other conditions & 38 & 8 & 2.39 & 0.97 & 1.58 & 3.74 & 31.69 \\
\hline Acute conditions & 7 & 1.47 & 2.29 & 0.71 & 3.57 & 4.00 & 12.86 \\
\hline Unknown conditions & 19 & 4 & 2.21 & 0.79 & 2.47 & 3.50 & 22.70 \\
\hline \multicolumn{8}{|l|}{ Age } \\
\hline$\sim 45$ & 109 & 22.95 & 2.66 & 1.11 & 3.20 & 3.76 & 34.05 \\
\hline $45 \sim$ & 233 & 49.05 & 2.48 & 1.21 & 2.14 & 4.00 & 25.29 \\
\hline $65 \sim$ & 133 & 28.00 & 2.65 & 1.11 & 1.85 & 3.92 & 35.86 \\
\hline \multicolumn{8}{|l|}{ Sex } \\
\hline Male & 139 & 29.26 & 2.51 & 1.13 & 1.99 & 3.91 & 22.21 \\
\hline Female & 336 & 70.74 & 2.59 & 1.17 & 2.44 & 3.93 & 33.41 \\
\hline \multicolumn{8}{|l|}{ Race } \\
\hline Others & 189 & 39.79 & 2.73 & 1.25 & 2.48 & 3.88 & 28.86 \\
\hline Non-Hispanic White & 286 & 60.21 & 2.46 & 1.09 & 2.19 & 3.95 & 30.88 \\
\hline \multicolumn{8}{|l|}{ Residence } \\
\hline Non-metropolitan & 67 & 14.11 & 2.71 & 1.16 & 2.26 & 3.98 & 29.49 \\
\hline Metropolitan & 408 & 85.89 & 2.55 & 1.16 & 2.31 & 3.91 & 30.22 \\
\hline \multicolumn{8}{|l|}{ Marital status } \\
\hline Unmarried & 187 & 39.37 & 2.64 & 1.20 & 2.85 & 4.08 & 34.41 \\
\hline Married & 288 & 60.63 & 2.53 & 1.13 & 1.96 & 3.82 & 27.31 \\
\hline
\end{tabular}




\begin{tabular}{|c|c|c|c|c|c|c|c|}
\hline Variables & Freq. & Percent & $\mathrm{SRH}^{*}$ & BMI* & $\begin{array}{l}\text { Psychological } \\
\text { distress }\end{array}$ & $\begin{array}{l}\text { Length of } \\
\text { caregiving* }\end{array}$ & $\begin{array}{l}\text { Caregiving } \\
\text { hours/week* }\end{array}$ \\
\hline Less than High S & 32 & 6.74 & 3.10 & 1.25 & 3.63 & 3.85 & 44.21 \\
\hline High School Grad & 66 & 13.89 & 2.83 & 1.20 & 2.72 & 3.75 & 41.86 \\
\hline Some College & 149 & 31.37 & 2.78 & 1.29 & 2.65 & 4.09 & 32.56 \\
\hline College Graduate & 228 & 48.00 & 2.29 & 1.05 & 1.79 & 3.87 & 24.83 \\
\hline \multicolumn{8}{|l|}{ Employment status } \\
\hline Unemployed & 226 & 47.88 & 2.75 & 1.18 & 2.94 & 3.97 & 41.44 \\
\hline Employed & 246 & 52.12 & 2.40 & 1.13 & 1.72 & 3.88 & 20.51 \\
\hline \multicolumn{8}{|l|}{ Income Ranges } \\
\hline$\$ 0$ to $\$ 9,999$ & 30 & 6.32 & 3.33 & 1.27 & 5.86 & 3.79 & 58.28 \\
\hline$\$ 10,000$ to $\$ 14,9$ & 22 & 4.63 & 3.11 & 1.27 & 3.33 & 4.30 & 40.00 \\
\hline$\$ 15,000$ to $\$ 19,9$ & 20 & 4.21 & 2.75 & 1.05 & 3.15 & 4.05 & 38.60 \\
\hline$\$ 20,000$ to $\$ 34,9$ & 66 & 13.89 & 2.65 & 1.12 & 2.78 & 3.71 & 38.50 \\
\hline$\$ 35,000$ to $\$ 49,9$ & 88 & 18.53 & 2.74 & 1.27 & 2.43 & 4.14 & 40.16 \\
\hline$\$ 50,000$ to $\$ 74,9$ & 67 & 14.11 & 2.54 & 1.12 & 1.81 & 4.09 & 22.29 \\
\hline$\$ 75,000$ to $\$ 99,9$ & 46 & 9.68 & 2.47 & 1.26 & 1.65 & 4.00 & 26.00 \\
\hline$\$ 100,000$ to $\$ 199$ & 99 & 20.84 & 2.24 & 1.05 & 1.43 & 3.80 & 20.79 \\
\hline$\$ 200,000$ or more & 37 & 7.79 & 2.11 & 1.08 & 1.35 & 3.54 & 13.95 \\
\hline \multicolumn{8}{|l|}{ Smoking } \\
\hline Current & 53 & 11.16 & 2.81 & 0.98 & 4.08 & 4.08 & 32.70 \\
\hline Former & 111 & 23.37 & 2.50 & 1.26 & 2.06 & 3.77 & 37.21 \\
\hline Never & 311 & 65.47 & 2.56 & 1.15 & 2.09 & 3.95 & 27.08 \\
\hline \multicolumn{8}{|l|}{ Drinking } \\
\hline Not & 292 & 61.47 & 2.69 & 1.21 & 2.29 & 3.94 & 32.91 \\
\hline Yes & 183 & 38.53 & 2.38 & 1.08 & 2.33 & 3.89 & 26.19 \\
\hline \multicolumn{8}{|c|}{$\begin{array}{l}\text { Recommended Physical } \\
\text { Activity }\end{array}$} \\
\hline Not & 324 & 68.21 & 2.65 & 1.21 & 2.44 & 3.96 & 31.08 \\
\hline Yes & 151 & 31.79 & 2.41 & 1.04 & 2.02 & 3.84 & 27.98 \\
\hline \multicolumn{8}{|c|}{ Having Health Insurance } \\
\hline Not & 34 & 7.16 & 2.69 & 0.88 & 3.06 & 4.15 & 54.38 \\
\hline Yes & 441 & 92.84 & 2.56 & 1.18 & 2.25 & 3.91 & 28.42 \\
\hline
\end{tabular}




\begin{tabular}{|lccccccc|}
\hline Variables & Freq. & Percent & SRH $^{*}$ & BMI* $^{*}$ & $\begin{array}{l}\text { Psychological } \\
\text { distress }\end{array}$ & $\begin{array}{l}\text { Length of } \\
\text { caregiving* }\end{array}$ & $\begin{array}{l}\text { Caregiving } \\
\text { hours/week }\end{array}$ \\
\hline Emotional Support & & & & & & & \\
\hline Never & 18 & 3.79 & 2.65 & 1.00 & 5.56 & 4.13 & 47.64 \\
\hline Rarely & 29 & 6.11 & 3.00 & 1.31 & 4.41 & 4.14 & 38.24 \\
\hline Sometimes & 80 & 16.84 & 2.79 & 1.21 & 3.00 & 3.91 & 36.00 \\
\hline Often & 103 & 21.68 & 2.59 & 1.17 & 2.34 & 3.96 & 29.34 \\
\hline Always & 245 & 51.58 & 2.43 & 1.13 & 1.59 & 3.87 & 26.60 \\
\hline *Mean; SRH = self-rated health; BMI = body mass index & & & & \\
\hline
\end{tabular}

\section{Discussion}

In this study, we aim to investigate the impact of patients' disease types on direct and indirect caregiving consequences, measured by caregiving time and caregiver burden, respectively. This is the first study that investigated the association between patients' disease types on caregiving time and caregiver burden, using data from a national survey that gauged a representative list of patients' disease types. Our study finds that compared with non-caregivers, caregivers experienced significantly worse levels of self-rated health, $\mathrm{BMI}$, and psychological distress. This finding resonates with what reported in previous studies [38, 43,44], and further highlights the need for intervention studies that could acknowledge and address caregiver burden. As approximately $21.3 \%$ of U.S. adults or 53.0 million Americans are informal caregivers who often do not have the adequate knowledge or skills needed to take care of the patients [18], whether or not successful interventions can deliver much-needed health solutions to these caregivers may impact not only the health of patients and caregivers, but also the health of the society $[45,46]$.

Adding insights to the literature [38, 47-49], findings of this study further suggest that caregivers who are female, older, had lower levels of education, physical inactivity, SES, unmarried, unemployed, not having health insurance experienced greater levels of caregiver burden. In other words, caregivers who belong to underserved communities are most likely to experience worse levels of caregiver burden. This worrying finding underscores the urgent need for timely and effective interventions that could alleviate these underserved populations' caregiver burden, such as intervention programs that are tailored to these caregivers' unique unmet needs [50-52]. As these disadvantaged caregivers are often difficult to reach via traditional intervention measures [53,54], researchers may consider using more agile and flexible programs, such as local reach programs [55-59] or technology-based solutions [60, 61], to deliver relevant and effective health solutions to these caregivers in a convenient and cost-effective fashion.

Results indicate that while caregivers of patients with neurological or related disease spent the greatest amount of time on caregiving, both in terms of overall caregiving duration and caregiving hours spent per week, their self-rated health, BMI status, and psychological stress are not the worst among all caregivers. Rather, caregivers of patients with aging-related disease or cancer reported worst self-rated health, caregivers of patients with orthopedic or related disease had the most worrisome BMI status, and caregivers of cancer patients experienced worst levels of psychological distress. In other words, medical conditions that require the greatest caregiving time may not exert the greatest levels of burden to caregivers. One way to explain this finding is that though some medical conditions may require a less alarming amount of caregiving time, the intensity and emotion strain of caregiving involved in taking care of the patients may be equally, if not more, burdensome $[18,28,31,32,62-64]$. While this finding helps shed 
light on the relationship between caregiving time and caregiver burden, it also emphasizes the need for more research to further chart factors that influence caregiving time and caregiver burden (e.g., disease severity [65]). To extend our understanding on how patients' disease characteristics influence caregiving consequences, future research could examine a more comprehensive list of disease-related characteristics to identify which factors have the greatest impact on caregiving consequences.

Based on findings of this study, researchers could also consider tailoring interventions based on patients and caregivers' needs and preferences [66-69]. Findings suggest though tailored and generic interventions both have potential to decrease caregivers' anxiety and depression symptoms, tailored interventions are more likely to induce sustained long-term health improvements in caregivers [66]. Evidence further indicates that dyad-based interventions can often take account of both patients' and caregivers' characteristics and preferences, and therefore have greater potential in improving patients and caregivers' coping skills and health outcomes than interventions designed solely for patients or caregivers [70-72]. Overall, findings of this study fill considerable gaps in the literature and offer critical insights that could help further the research field.

\section{Limitations}

First, the survey was cross-sectional in nature, which limits causal inference. We adopted a validated and reliable nationally representative survey, HINTS 5 Cycle 2, as our data source. While there are many benefits in secondary data analysis of a nationally representative survey (e.g., cost-effective, time-efficient, and ability to generate findings with greater replicability and comparability) [73-75], as the survey is not specifically designed for this study, substantial research rigor could be lost due to the lack of purposeful survey design. To address these drawbacks, future research could develop research with tailored and robust survey design (e.g., longitudinal or mixed-methods) to increase research rigor.

\section{Conclusion}

This is the first study that investigated the impact of patients' disease types on caregiving time and caregiver burden, using a comprehensive list of disease types surveyed in a nationally representative survey. Findings of this study show that underserved caregiver populations often subject to greater levels of caregiver burden in terms of self-rated health, BMI, and psychological distress. Results also indicate that great variations are found in the impact of patients' disease types on caregiving time and caregiver burden. These insights extend our understanding towards the relationship between patients' disease types and caregiving consequences. Furthermore, findings of this study also underscore the need for healthcare researchers to adopt a nuanced approach in acknowledging and addressing caregiver burden, such as tailoring interventions based on patients and caregivers' needs and preferences. Overall, our study fills important gaps in the literature and offers great opportunities for better understanding the interaction between patients' disease characteristics and caregivers' burden and health outcomes.

\section{Declarations}

\section{Ethics approval and consent to participate}

Not applicable.

\section{Consent for publication}

Not applicable. 


\section{Competing interests}

None.

\section{Funding}

None

\section{Authors' contributions}

ZS developed the research idea, oversaw the data analysis, and wrote the manuscript. ZZ conducted the data analysis, wrote the results section, and reviewed the manuscript. JG reviewed and edited the manuscript. All three authors participated in the process of building of the manuscript.

\section{Acknowledgements}

We wish to express our gratitude to the editors and reviewers for their constructive input.

\section{References}

1. Allen, A.P., et al., Informal caregiving for dementia patients: the contribution of patient characteristics and behaviours to caregiver burden. Age and Ageing, 2019. 49(1): p. 52-56.

2. Johansen, S., M. Cvancarova, and C. Ruland, The effect of cancer patients' and their family caregivers' physical and emotional symptoms on caregiver burden. Cancer Nursing, 2018. 41(2).

3. Shrestha, D., et al., Factors affecting psychological burden on the informal caregiver of patients with cirrhosis: Looking beyond the patient. Journal of Clinical and Experimental Hepatology, 2020. 10(1): p. 9-16.

4. Khalaila, R., Caregiver burden and compassion fatigue among Arab family caregivers of older relatives. Journal of Applied Gerontology, 2020: p. 0733464820920100.

5. Saban, K.L., et al., Perceived health, caregiver burden, and quality of life in women partners providing care to veterans with traumatic brain injury. J Rehabil Res Dev, 2016. 53(6): p. 681-692.

6. Simón, M.A., et al., Caregiver burden and sleep quality in dependent people's family caregivers. J Clin Med, 2019. 8(7).

7. Bártolo, A., et al., Effectiveness of psycho-educational interventions with telecommunication technologies on emotional distress and quality of life of adult cancer patients: a systematic review. Disability and Rehabilitation, 2019. 41(8): p. 870-878.

8. Hansen, B., et al., Affiliate stigma and caregiver burden in intractable epilepsy. Epilepsy Behav, 2018. 85: p. 1-6.

9. Su, J.-A. and C.-C. Chang, Association between family caregiver burden and affiliate stigma in the families of people with dementia. International Journal of Environmental Research and Public Health, 2020. 17(8): p. 2772.

10. Tyo, M.B. and M.K. McCurry, An integrative review of measuring caregiver burden in substance use disorder. Nurs Res, 2020.

11. Lou, Q., et al., Comprehensive analysis of patient and caregiver predictors for caregiver burden, anxiety and depression in Alzheimer's disease. J Clin Nurs, 2015. 24(17-18): p. 2668-78.

12. Zhong, Y., J. Wang, and S. Nicholas, Social support and depressive symptoms among family caregivers of older people with disabilities in four provinces of urban China: the mediating role of caregiver burden. BMC Geriatr, 2020. 20(1): p. 3. 
13. França, A.B., et al., Symptoms of mood disorders in family carers of older people with dementia who experience caregiver burden: a network approach. Age Ageing, 2020.

14. Noguchi, T., et al., The association between family caregiver burden and subjective well-being and the moderating effect of social participation among Japanese adults: A cross-sectional study. Healthcare (Basel), 2020. 8(2).

15. Lu, N., J. Liu, and V.W. Lou, Caring for frail elders with musculoskeletal conditions and family caregivers' subjective well-being: The role of multidimensional caregiver burden. Arch Gerontol Geriatr, 2015. 61(3): p. 411-8.

16. Hsiao, C.Y., H.L. Lu, and Y.F. Tsai, Caregiver burden and health-related quality of life among primary family caregivers of individuals with schizophrenia: a cross-sectional study. Qual Life Res, 2020.

17. Tulek, Z., et al., Caregiver burden, quality of life and related factors in family caregivers of dementia patients in Turkey. Issues Ment Health Nurs, 2020: p. 1-9.

18. American Association of Retired Persons, Caregiving in the U.S. 2020.

19. Schrank, B., et al., Gender differences in caregiver burden and its determinants in family members of terminally ill cancer patients. Psycho-Oncology, 2016. 25(7): p. 808-814.

20. Park, J., et al., Self-efficacy and social support for psychological well-being of family caregivers of care recipients with dementia with Lewy bodies, Parkinson's disease dementia, or Alzheimer's disease. Social Work in Mental Health, 2019. 17(3): p. 253-278.

21. Monin, J.K., T.D. Jorgensen, and J.L. MacNeil Vroomen, Self-reports and caregivers' proxy reports of unmet needs of persons with dementia: Implications for both partners' health-related quality of life. The American Journal of Geriatric Psychiatry, 2020. 28(3): p. 363-367.

22. Koopman, E., et al., Factors associated with caregiver burden among adult (19-64 years) informal caregivers An analysis from Dutch Municipal Health Service data. Health \& Social Care in the Community, 2020. n/a(n/a).

23. Seo, Y.J. and H. Park, Factors influencing caregiver burden in families of hospitalised patients with lung cancer. Journal of Clinical Nursing, 2019. 28(9-10): p. 1979-1989.

24. Boztepe, H., et al., Predictors of caregiver burden in mothers of children with leukemia and cerebral palsy. Journal of Psychosocial Oncology, 2018: p. 1-10.

25. Sagut, P. and Z. Çetinkaya Duman, Comparison of caregiver burden in first episode versus chronic psychosis. Archives of Psychiatric Nursing, 2016. 30(6): p. 768-773.

26. Avşar, U., et al., Caregiver burden, anxiety, depression, and sleep quality differences in caregivers of hemodialysis patients compared with renal transplant patients. Transplantation Proceedings, 2015. 47(5): p. 1388-1391.

27. Elmståhl, S., et al., The association between medical diagnosis and caregiver burden: A cross-sectional study of recipients of informal support and caregivers from the general population study 'Good Aging in Skåne', Sweden. Aging Clinical and Experimental Research, 2018. 30(9): p. 1023-1032.

28. Liu, S., et al., Caregiver burden, sleep quality, depression, and anxiety in dementia caregivers: A comparison of frontotemporal lobar degeneration, dementia with Lewy bodies, and Alzheimer's disease. International Psychogeriatrics, 2018. 30(8): p. 1131-1138.

29. Svendsboe, E., et al., Caregiver burden in family carers of people with dementia with Lewy bodies and Alzheimer's disease. Int J Geriatr Psychiatry, 2016. 31(9): p. 1075-83.

30. Branger, C., et al., Variance in caregiver burden predicted by patient behaviors versus neuropsychological profile. Applied Neuropsychology: Adult, 2018. 25(5): p. 441-447. 
31. Küçükgüçlü, Ö., et al., Examining factors affecting caregiver burden: A comparison of frontotemporal dementia and Alzheimer's disease. American Journal of Alzheimer's Disease \& Other Dementiasr, 2017. 32(4): p. 200-206.

32. Uflacker, A., et al., Caregiver burden in atypical dementias: Comparing frontotemporal dementia, CreutzfeldtJakob disease, and Alzheimer's disease. International Psychogeriatrics, 2016. 28(2): p. 269-273.

33. Nelson, D., et al., The Health Information National Trends Survey (HINTS): Development, design, and dissemination. Journal of Health Communication, 2004. 9(5): p. 443-460.

34. National Cancer Institute. Health Information National Trends Survey 5 Cycle 2 methodology report. 2018; Available from: https://hints.cancer.gov/docs/methodologyreports HINTS5_Cycle_2_Methodology_Report.pdf.

35. Shilling, V., et al., Patient-reported outcome measures for cancer caregivers: A systematic review. Quality of Life Research, 2016. 25(8): p. 1859-1876.

36. Bastawrous, M., Caregiver burden-A critical discussion. International Journal of Nursing Studies, 2013. 50(3): p. 431-441.

37. Ory, M.G., et al., Prevalence and impact of caregiving: A detailed comparison between dementia and nondementia caregivers. The Gerontologist, 1999. 39(2): p. 177-186.

38. Shaffer, K.M., et al., Informal caregivers' use of internet-based health resources: An analysis of the health information national trends survey. JMIR Aging, 2018. 1(2): p. e11051.

39. Centers for Disease Control and Prevention. About adult BMI. 2020 [cited 2020 June 10]; Available from: https://www.cdc.gov/healthyweight/assessing/bmi/adult_bmi/index.html.

40. Löwe, B., et al., A 4-item measure of depression and anxiety: validation and standardization of the Patient Health Questionnaire-4 (PHQ-4) in the general population. J Affect Disord, 2010. 122(1-2): p. 86-95.

41. U.S. Department of Agriculture. Rural-urban continuum codes. US Department of Agriculture Economic Research Service 2013 [cited 2020 June 10]; Available from: https://www.ers.usda.gov/data-products/rural-urbancontinuum-codes.aspx.

42. StataCorp, Stata statistical software: Release 14.1. 2016, College Station, TX.

43. Barnhart, W.R., et al., Caregiving in the shadows: National analysis of health outcomes and intensity and duration of care among those who care for people with mental illness and for people with developmental disabilities. Disability and Health Journal, 2020. 13(1): p. 100837.

44. Berry, H.G., et al., Health characteristics and outcomes of caregivers in the United States: An Analysis of the 2017 Health Information National Trends Survey (HINTS). Disability and Health Journal, 2020. 13(1): p. 100821.

45. Schulz, R., et al., Economic impact of family caregiving, in Families Caring for an Aging America. 2016, National Academies Press (US).

46. Oliva-Moreno, J., et al., The economic value of time of informal care and its determinants (The CUIDARSE Study). PloS one, 2019. 14(5): p. e0217016-e0217016.

47. Mollica, M.A., A.W. Smith, and E.E. Kent, Caregiving tasks and unmet supportive care needs of family caregivers: A U.S. population-based study. Patient Education and Counseling, 2020. 103(3): p. 626-634.

48. Edwards, V.J., et al., Characteristics and health status of informal unpaid caregivers - 44 States, District of Columbia, and Puerto Rico, 2015-2017. Morbidity and Mortality Weekly Report, 2020. 69(7): p. 183-188.

49. Bonacasa, M.D., et al., A translational approach to design effective intervention tools for informal caregivers of dependent cancer patients. Public Health, 2019. 168: p. 50-58.

50. Lambert, S.D. and A. Girgis, Unmet supportive care needs among informal caregivers of patients with cancer: Opportunities and challenges in informing the development of interventions. Asia-Pacific Journal of Oncology

Page $18 / 20$ 
Nursing, 2017. 4(2): p. 136-139.

51. Rexhaj, S., et al., Feasibility and accessibility of a tailored intervention for informal caregivers of people with severe psychiatric disorders: A pilot study. Frontiers in Psychiatry, 2017. 8(178).

52. Shaw, C.A., et al., Effects of a video-based intervention on caregiver confidence for managing dementia care challenges: Findings from the FamTechCare clinical trial. Clinical Gerontologist, 2020: p. 1-10.

53. Goldman, $\mathrm{H}$., et al., Recruitment and retention of the hardest-to-reach families in community-based asthma interventions. Clinical Trials, 2018. 15(6): p. 543-550.

54. Haley, S.J., et al., Barriers and strategies for recruitment of racial and ethnic minorities: Perspectives from neurological clinical research coordinators. Journal of Racial and Ethnic Health Disparities, 2017. 4(6): p. 12251236.

55. Richardson, V.E., et al., At the intersection of culture: Ethnically diverse dementia caregivers' service use. Dementia, 2017. 18(5): p. 1790-1809.

56. Gelman, C.R., Learning From recruitment challenges: Barriers to diagnosis, treatment, and research participation for Latinos with symptoms of Alzheimer's disease. Journal of Gerontological Social Work, 2010. 53(1): p. 94113.

57. McLean, K., et al., Foster and kinship carer experiences of accessing healthcare: A qualitative study of barriers, enablers and potential solutions. Children and Youth Services Review, 2020. 113: p. 104976.

58. Griffith, R., et al., Strategies for longitudinal recruitment and retention with parents and preschoolers exposed to significant adversity: The PAIR project as an example of methods, obstacles, and recommendations. Children and Youth Services Review, 2020. 110: p. 104770.

59. Kajiyama, B., et al., Helping Hispanic dementia caregivers cope with stress using technology-based resources. Clinical Gerontologist, 2018. 41(3): p. 209-216.

60. Wozney, L., et al., Facebook ads to the rescue? Recruiting a hard to reach population into an Internet-based behavioral health intervention trial. Internet Interventions, 2019. 17: p. 100246.

61. Ryan-Pettes, S.R., L.L. Lange, and K.I. Magnuson, Mobile phone access and preference for technology-assisted aftercare among low-income caregivers of teens enrolled in outpatient substance use treatment: Questionnaire study. JMIR Mhealth Uhealth, 2019. 7(9): p. e12407.

62. Kronenberg, L.M., et al., Burden and expressed emotion of caregivers in cases of adult substance use disorder with and without Attention Deficit/Hyperactivity Disorder or Autism Spectrum Disorder. International Journal of Mental Health and Addiction, 2016. 14(1): p. 49-63.

63. Martinez, M., et al., Emotion detection deficits and decreased empathy in patients with Alzheimer's disease and Parkinson's disease affect caregiver mood and burden. Frontiers in Aging Neuroscience, 2018. 10(120).

64. Chang, C.-C., et al., Comparing affiliate stigma between family caregivers of people with different severe mental illness in Taiwan. The Journal of Nervous and Mental Disease, 2017. 205(7).

65. McCarron, M., et al., Alzheimer's dementia in persons with Down's syndrome: Predicting time spent on day-to-day caregiving. Dementia, 2005. 4(4): p. 521-538.

66. Márquez-González, M., et al., Tailored versus manualized interventions for dementia caregivers: The functional analysis-guided modular intervention. Psychology and Aging, 2020. 35(1): p. 41-54.

67. de Oliveira, A.M., et al., An intervention to reduce neuropsychiatric symptoms and caregiver burden in dementia: Preliminary results from a randomized trial of the tailored activity program-outpatient version. International Journal of Geriatric Psychiatry, 2019. 34(9): p. 1301-1307. 
68. lio, M., et al., Tailored education to increase self-efficacy for caregivers of children with asthma: A randomized controlled trial. Computers, Informatics, Nursing, 2017. 35(1).

69. El-Jawahri, A., et al., Multimodal psychosocial intervention for family caregivers of patients undergoing hematopoietic stem cell transplantation: A randomized clinical trial. Cancer, 2020. 126(8): p. 1758-1765.

70. Badger, T.A., et al., Randomized controlled trial of supportive care interventions to manage psychological distress and symptoms in Latinas with breast cancer and their informal caregivers. Psychology \& Health, 2020. 35(1): p. 87-106.

71. Milbury, K., et al., Pilot randomized, controlled trial of a dyadic yoga program for glioma patients undergoing radiotherapy and their family caregivers. Neuro-Oncology Practice, 2018. 6(4): p. 311-320.

72. von Heymann-Horan, A., et al., Dyadic coping in specialized palliative care intervention for patients with advanced cancer and their caregivers: Effects and mediation in a randomized controlled trial. Psycho-Oncology, 2019. 28(2): p. 264-270.

73. Kiecolt, K.J., et al., Secondary analysis of survey data. Vol. 53. 1985, Newbury Park, CA: Sage.

74. Lawton, C., Effective use of secondary quantitative data sources, in Handbook of research methods on the quality of working lives, D. Wheatley, Editor. 2019, Edward Elgar Publishing: Cheltenham, UK. p. 177-193.

75. Walliman, N., Research methdos the basics. 2011, New York: NY: Routledge. 